\title{
Space Weather Research by means of High Mountain Alma-Ata Neutron Monitor
}

\author{
A.V. Belov ${ }^{1}$, V.I. Drobzhev ${ }^{2}$, E.A.Eroshenko ${ }^{1}$, O. N. Kryakunova ${ }^{2}$, \\ N.F. Nikolaevskiy ${ }^{2}$, V.G.Yanke ${ }^{1}$, Zh.Sh.Zhantaev ${ }^{2}$ \\ ${ }^{1}$ Institute of Terrestrial Magnetism, Ionosphere and Radio Wave Propagation RAS, 142190, \\ Troitsk, Moscow region, Russia \\ ${ }^{2}$ Institute of Ionosphere, Ministry of Education and Science, 480020, Alma-Ata, Kazakhstan \\ email: krolganik@yandex.ru
}

For multi-wavelength investigations of solar activity it may be used the intensity of cosmic ray, registered by means of neutron monitors, because the variations of cosmic ray intensity are very sensitive for local and global solar activity changes. It is important also for space weather investigation. So, we use special indices characterized the level of cosmic ray activity and some peculiarities of the space weather (Belov et al. 1999). To apply the approach, already used for cosmic ray activity calculation, to the single detector data, we have to study daily run of the counting rate. During quiet days we have in the neutron monitor data sinuslike daily variation. The characteristics of the normal for the current period anisotropy and location of the station define it. Thus, on Alma-Ata station counting rate mainly increases on the first half of day and decreases on the second one. The density changing and the anomalous anisotropy behavior lead to distortion of daily run, and the greater is of cosmic ray disturbance, the more is distortion. Let in hour t the variation of the counting rate relatively to daily mean is $\Delta(t)$, and its hourly change is $d(t)=\Delta(t)-\Delta(t-1)$. Comparing this value with the same for the quiet period $d_{0}(t)$, we can find hourly indices of cosmic ray disturbance for the single station (for high altitude Alma-Ata station- $\left.C R A_{a a t b}\right)$ as:

$$
C R A_{\text {aatb }}=b\left|d(t)-d_{0}(t)\right| s^{-1}-a .
$$

The problem of the random variations on using indices from a single station becomes more pressing than for global CRA-indices. The use of cosmic ray detector with high counting rate as high altitude Alma-Ata neutron monitor is preferable here. The AlmaAta high altitude neutron monitor has a favorable location and very good statistics (1200 counts per second) to detect different cosmic ray effects conditioned by the solar activity and space weather. The combination of geomagnetic cut-off rigidity (6.7 GV) and high altitude (3340 $\mathrm{m}$ above sea level) makes our station enable to record ground level enhancements (GLE) for the events where maximal rigidity of the protons exceeds 6.7 GV. CRA indices for Alma-Ata station were calculated with $s=0.09 \%, b=3, a=1$. The local cosmic ray activity indices (CRA) were calculated for every hour within the period of 1971-1994 on the base of cosmic ray density, obtained by means of high mountain Alma-Ata neutron monitor. The behavior of cosmic ray activity indices reveals not only the greatest cosmic ray disturbances but the smaller cosmic ray variation as well, because these indices are sensitive to heliospheric effects (Figure 1-the left plot). The data from a single neutron monitor has a decisive advantage: these data allow estimating of cosmic ray disturbance in the real time. In order to analyse interplanetary and geo-space situation during of dangerous periods of space environment we use real-time observations of cosmic ray intensity in 1-minute regime of accumulation. Besides that we calculate hour cosmic ray activity indices in real time. Observations of energetic particles and galactic 
cosmic ray variations in real time recorded by Alma-Ata neutron monitor may be applied to forecasting purposes also (http://tien-shan.uni.sci.kz). Using complex database of satellite malfunctions we analysed the connection between malfunction frequency (malfunction number per active satellite) and cosmic ray activity indices. Malfunction data on different satellites in the period 1971-1994 were analyzed in the search of possible influence of different space environmental parameters. The database was created, which combined, beyond the malfunction information, various characteristics of space weather: solar activity indices, geomagnetic activity indices, fluxes and fluencies of electrons and protons at different energy, high energy cosmic ray variations, solar wind characteristics and other interplanetary and geophysical data (Belov et al. 2003, 2004). From one side, these indices behave themselves similar to the geomagnetic activity indices, since the Forbush effects and geomagnetic storms are caused by the common reasons and occurred simultaneously as a rule. From the other side, they respond to the proton enhancements. Thus, in the CRA-indices two different classes of the satellite malfunctions reasons may be revealed. It was shown that cosmic ray activity indices seem to be useful as one of characteristics of the disturbed periods of space weather, connected with satellite malfunctions and Alma-Ata high mountain cosmic ray station is one of the most suitable and sensitive stations for investigation and forecasting of dangerous situation of space weather (Figure 1-the right plot).
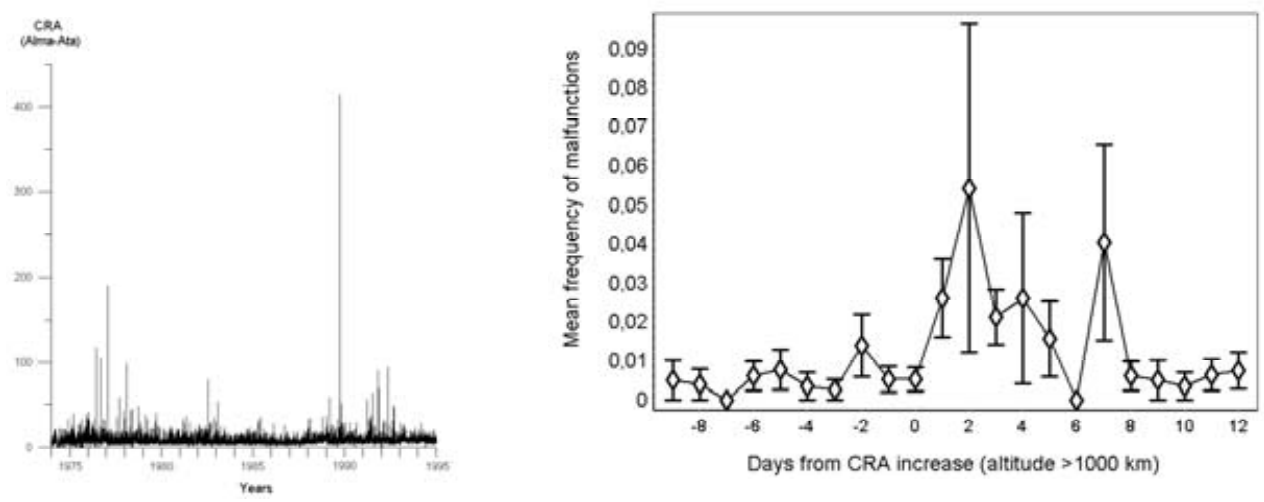

Figure 1. CRA indices for Alma-Ata station through 1974-1994 and the averaged satellite malfunction frequency

\section{References}

Belov, A.V., Eroshenko, E.A., Yanke, V.G. \& Kryakunova, O.N. 1999 Proc. 26th ICRC 6, 472-475.

Belov, A. et al 2003a Proc. 28th ICRC 1, 4213-4216.

Belov, A. et al. 2004 In Effects of Space Weather on Technology Infrastructure (ed. I. A. Daglis), NATO Science Series, vol. 176, 147-163. 